CONCISE REPORT

\title{
Inhibition of sumoylation prevents experimental fibrosis
}

\author{
Aisa Khodzhigorova, ${ }^{1}$ Alfiya Distler, ${ }^{1}$ Veronika Lang, ${ }^{1}$ Clara Dees, ${ }^{1}$ Holm Schneider, ${ }^{2}$ \\ Christian Beyer, ${ }^{1}$ Kolja Gelse, ${ }^{3}$ Oliver Distler, ${ }^{4}$ Georg Schett, ${ }^{1}$ Jörg H W Distler ${ }^{1}$
}

${ }^{1}$ Department of Internal Medicine III and Institute for Clinical Immunology, University of Erlangen-Nuremberg, Erlangen, Germany ${ }^{2}$ Department of Pediatrics, University of Erlangen-

Nuremberg, Erlangen, Germany ${ }^{3}$ Department of Surgery, University of ErlangenNuremberg, Erlangen, Germany ${ }^{4}$ Center of Experimental Rheumatology and Zurich Center of Integrative Human Physiology, University Hospital Zurich, Zurich, Switzerland

\section{Correspondence to}

Jörg H W Distler, Department of Internal Medicine 3 and Institute for Clinical Immunology, University of Erlangen-Nuremberg, Erlangen D-91054, Germany; joerg. distler@uk-erlangen.de

Received 23 March 2012 Accepted 19 July 2012

\begin{abstract}
Objectives Fibrosis is a predominant cause of death in systemic sclerosis (SSc). First epigenetic modifications have recently been shown to contribute to activation of SSc fibroblasts. Here, we investigated inhibition of sumoylation as a novel antifibrotic approach.

Methods Sumoylation was inhibited by siRNA-mediated knockdown of the Small Ubiquitin-like MOdifiers (SUMO) E2-conjugating enzyme Ubc9, which is essential for sumoylation. The effects of knockdown of Ubc9 were analysed in bleomycin-induced dermal fibrosis, and in the model of fibrosis induced by overexpression of a constitutively active TGF-beta receptor type I (TBR). SUM0-1 and phosphorylated Smad3 were detected by immunohistochemistry.

Results Increased staining for SUMO-1 was detected in patients with SSc and in experimental fibrosis. Inhibition of sumoylation exerted potent antifibrotic effects and prevented dermal thickening, myofibroblast differentiation and accumulation of collagen induced by bleomycin, or by overexpression of constitutively active TBR. Moreover, knockdown of Ubc9 reduced the accumulation of phosphorylated Smad3 in experimental fibrosis indicating that inhibition of sumoylation may normalise canonical TGF- $\beta$ signalling in vivo.

Conclusions We demonstrate that inhibition of sumoylation reduces canonical TGF- $\beta$ signalling and prevents experimental fibrosis in different preclinical models. These data provide first evidence that targeting of aberrant sumoylation may be a novel therapeutic approach for fibrotic diseases.
\end{abstract}

\section{INTRODUCTION}

Systemic sclerosis (SSc) is characterised by progressive accumulation of extracellular matrix. ${ }^{1}$ The resulting tissue fibrosis is a major cause of the high morbidity and lethality in SSc patients. ${ }^{2}$ However, the molecular mechanisms of the aberrant activation of SSc fibroblasts are only partially understood.

Post-translational modifications of protein are important regulators of cell signalling that regulate enzymatic activity, protein stability or subcellular localisation. ${ }^{3}$ Among these post-translational modifications is sumoylation. By contrast with other common protein modifications, such as phosphorylation, only a rather limited spectrum of substrates for sumoylation has been identified. ${ }^{4}$ Sumoylation describes the covalent attachment of Small Ubiquitin-like MOdifiers (SUMO) to lysine residues of target proteins. ${ }^{5}$ Although the three- dimensional structures of SUMO proteins are similar to that of ubiquitin, and sumoylation is directed by an enzymatic cascade analogous to that mediating ubiquitination, the functional consequences of ubiquitination and sumoylation differ. Whereas ubiquitination rather exclusively marks proteins for proteasomal degradation, the effects of sumoylation are more diverse, and also alter the nuclear-cytosolic transport and the transcriptional activity in addition to protein stability. ${ }^{6}$ Besides modifying the activity of individual transcription factors, sumoylation of histones provides an additional epigenetic mechanism for SUMO proteins to modify cellular pathways. ${ }^{7}$ Given (1) very recent findings that sumoylation regulates the catalytic activity of topoisomerase I in SSc as first evidence for a role of sumylation in the pathogenesis of SSc, ${ }^{8}$ (2) reports on the regulation of canonical Smad signalling by sumoylation and (3) the importance of epigenetics in SSc, ${ }^{9-12}$ we hypothesised that aberrant sumoylation may play a role for the development of fibrosis in SSc.

\section{MATERIAL AND METHODS \\ Patients}

Skin biopsies were obtained from skin of 22 SSc patients and from 13 healthy volunteers matched for age and sex. All SSc patients fulfilled the LeRoy criteria for SSc. ${ }^{13}$ The median age of SSc patients was 43 years (range 18-64 years), and their median disease duration was 5 years (range 1-17 years); eight had limited cutaneous disease and 14 had diffuse cutaneous SSc. Four patients received MTX at the time of biopsy and four additional patients were treated with MMF. All patients and controls signed a consent form approved by the local institutional review boards.

\section{In vivo transfection with siRNAs against Smo in bleomycin-induced skin fibrosis}

Complexes of siRNA and atelocollagen (Koken, Tokyo, Japan) were prepared as described. ${ }^{14}$ siRNA Duplexes (sense 5'-CAAUGAACCUGAUGAACUGUU-3', antisense 5'-PCAGUUCAUCAGGUUCAUUGUU-3') from Dharmacon (Lafayette, Colorado, USA) were used. Non-targeting siRNA duplexes served as controls. Atelocollagen/siRNA complexes were injected intracutaneously once weekly.

\section{Mouse models of fibrosis}

Bleomycin-induced dermal fibrosis was induced according to established protocols. ${ }^{15}$ 
Dermal fibrosis induced by adenoviral overexpression of a constitutively active TGF- $\beta$ receptor I (AdTBR) was performed as described. ${ }^{16}$ Briefly, $6.67 \times 10^{7}$ infectious units of replicationdeficient type $V$ adenoviruses overexpressing TBR were injected intradermally. Mice injected with adenoviruses carrying a LacZ reporter gene served as controls.

Treatment with siRNA was initiated together with the injections of bleomycin or AdTBR, respectively.

\section{Analysis of murine skin}

Dermal thickness and hydroxyproline content were analysed as described previously. ${ }^{17} 18$

\section{Staining for SUM0-1, $\alpha$-smooth muscle actin and pSmad3}

The expression of SUMO-1 was detected by staining with rabbit monoclonal antibodies (dilution 1:250, 1563-1, Epitomics, Burlingame, California) overnight at $4^{\circ} \mathrm{C}$, polyclonal goat antirabbit antibodies (Jackson ImmunoResearch, Soham, UK) labelled with horseradish peroxidase and 3,3' diaminobenzidine tetrahdydrochloride (DAB, Merck, Darmstadt, Germany). The expression levels were quantified as follows: $0=$ no expression, $1=$ low intensity staining in $<50 \%$ of cells, $2=$ intense staining in $<50 \%$ of cells, or less intense staining in $>50 \%$ of cells, $3=$ intense staining in $>50 \%$ of cells.

Stainings for $\alpha \mathrm{SMA}$ ( $\alpha \mathrm{SMA}$ antibodies: clone 1A4, Sigma-Aldrich, Steinheim, Germany) and for pSmad3 (pSmad3 antibodies: Cell Signalling, Danvers, Massachusetts, USA), were performed as described. ${ }^{15}$

\section{Statistical analysis}

Data are expressed as the median $\pm I O R$. The Wilcoxon signed rank test for related samples, and the Mann-Whitney $U$ test were used for statistical analyses. p Values less than 0.05 were considered significant.

\section{RESULTS \\ Increased sumoylation with enhanced expression of SUM0-1 in SSc}

To determine to extend of sumoylation in SSc, we stained for SUMO-1, the most abundant sumoylation tag. The expression of SUMO-1 was extensive in fibrotic skin of SSc patients with prominent staining in fibroblasts, endothelial cells and keratinocytes. No differences were detected between limited cutaneous and diffuse cutaneous SSc. We also did not observe correlations between the expression of SUMO-1 and disease duration. The expression of SUMO-1 in non-lesional skin was comparable with that in lesional skin. Moreover, the expression levels of SUMO-1 did not differ between SSc patients treated with MTX or MMF and SSc patients without potential DMARDs. By contrast with the broad staining in SSc, SUMO-1 was detected only in some fibroblasts and endothelial cells as well as a subset of keratinocytes in the skin of healthy individuals (figure 1A). The expression of SUMO-1 was also upregulated in murine models of SSc. The expression patterns in bleomycin-induced dermal fibrosis and in TBR-driven fibrosis resembled those of SSc patients with intense staining of fibroblasts, endothelial cells and keratinocytes (figure 1B,C).

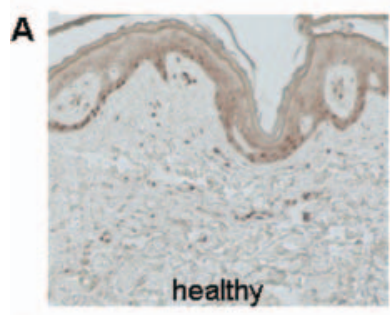

B

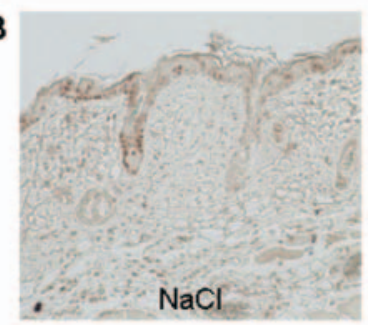

C

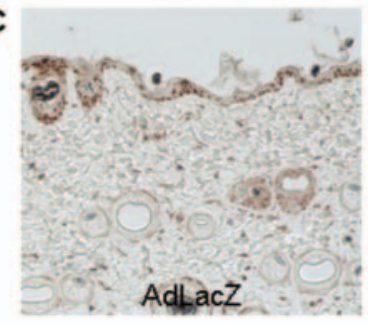

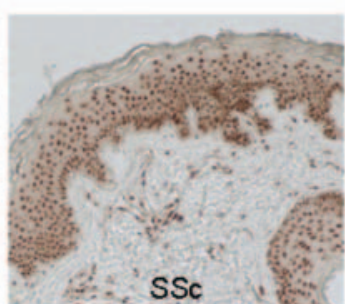
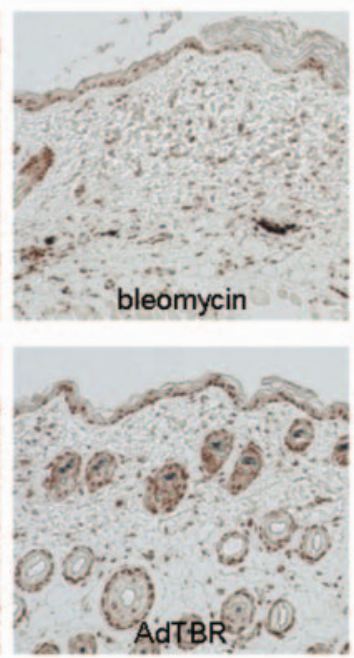
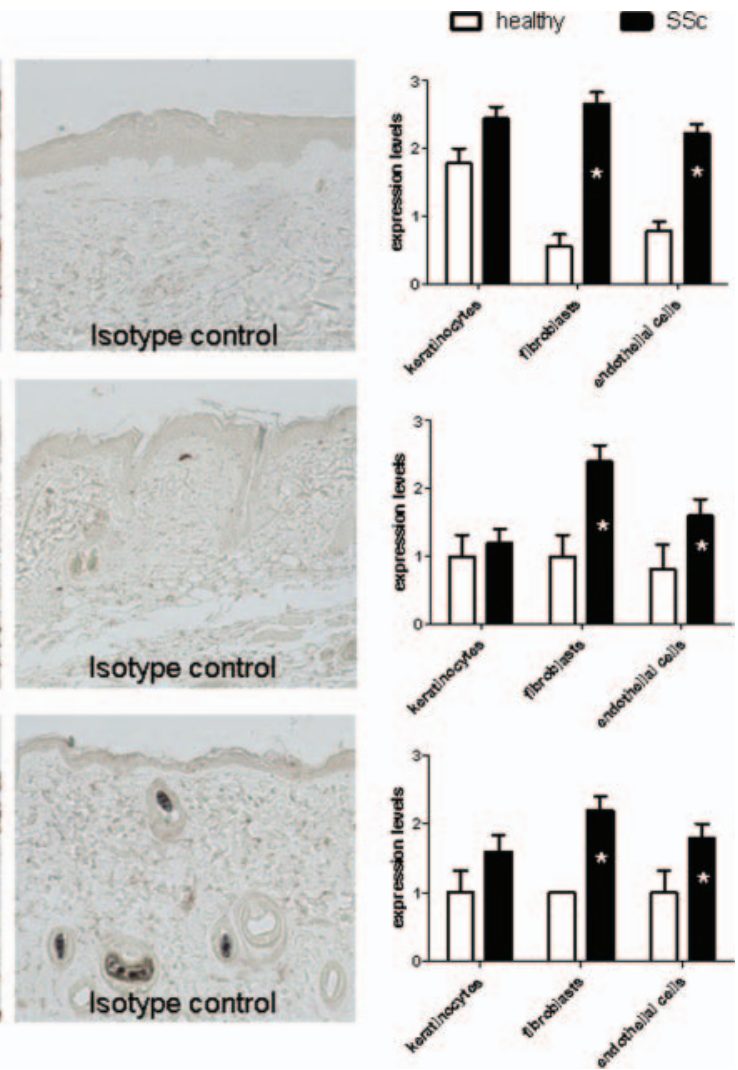

Figure 1 Sumoylation is increased in systemic sclerosis (SSc) and in experimental models of fibrosis. (A) Immunohistochemistry demonstrates increased staining for Small Ubiquitin-like MOdifiers (SUMO)-1 in SSc patients compared with healthy individuals with prominent staining in fibroblasts, endothelial cells and keratinocytes. SUMO-1 is also increased in bleomycin-induced dermal fibrosis (B) and in TGF- $\beta$ receptor-mediated fibrosis (C) compared with non-fibrotic control mice. Representative images of human sections are shown at 200 -fold magnification, murine sections at 100 -fold magnification. Semiquantitative analyses of the expression levels of SUMO-1 are shown on the right. ${ }^{*} p<0.05$, as compared with non-fibrotic controls. 


\section{Knockdown of Ubc9 prevents bleomycin-induced fibrosis}

To investigate whether increased sumoylation contributes to the development of fibrosis, we inhibited sumoylation by siRNA-mediated knockdown of the SUMO E2-conjugating enzyme Ubc9, which is essential for sumoylation. ${ }^{4}$ Knockdown of Ubc9 protected from bleomycin-induced dermal fibrosis (figure 2A). Dermal thickening was reduced by $75 \%$ in bleomycin-challenged mice injected with siRNA against Ubc9 compared with bleomycin-treated mice injected with nontargeting control siRNA $(p=0.01)$ (figure $2 B$ ). Inhibition of sumoylation also significantly reduced the hydroxyproline content and the number of myofibroblasts almost back to the levels of control mice injected with $\mathrm{NaCl}$ (figure 2C,D). Moreover, knockdown of Ubc9 reduced the nuclear accumulation of pSmad3 upon bleomycin challenge by $82 \%$ compared with bleomycin-treated mice injected with control siRNA $(p=0.03)$ (figure $2 \mathrm{E}$ ).

\section{Inhibition of sumoylation prevents TBR-induced fibrosis}

After demonstrating that inhibition of sumoylation inhibits fibrosis in an inflammatory model resembling early stages of SSc, we next aimed to investigate whether Ubc9 may also be a target in a less inflammatory model resembling later stages of
SSc with endogenous activation of fibroblasts. siRNA Against Ubc9 potently reduced the development of fibrosis (figure $3 \mathrm{~A}$ ). Dermal thickening, accumulation of collagen and differentiation of resting fibroblasts into myofibroblasts were all significantly reduced in TBR-induced fibrosis upon knockdown of Ubc9 (figure 3B-D). The dermal thickness, the hydroxyproline content and the myofibroblast counts were comparable with those of non-fibrotic LacZ control mice demonstrating almost complete prevention of fibrosis. Inhibition of sumoylation also decreased canonical Smad signalling and prevented nuclear accumulation of pSmad 3 (figure $3 \mathrm{E}$ ).

\section{DISCUSSION}

Selective modulations of other protein modifications have not broadly emerged into clinical therapies. In particular, inhibition of protein sumoylation has not yet been evaluated for therapeutic purposes. We identified a potential therapeutic implication for inhibiting sumoylation. Knockdown of the SUMO E2-conjugating enzyme Ubc9, which selectively prevents sumoylation, exerted potent antifibrotic effects in a preclinical model resembling early and late stages of SSc. Knockdown of Ubc9 prevented fibrosis induced by overexpression of a constitutively active TBR construct, indicating that inhibition of
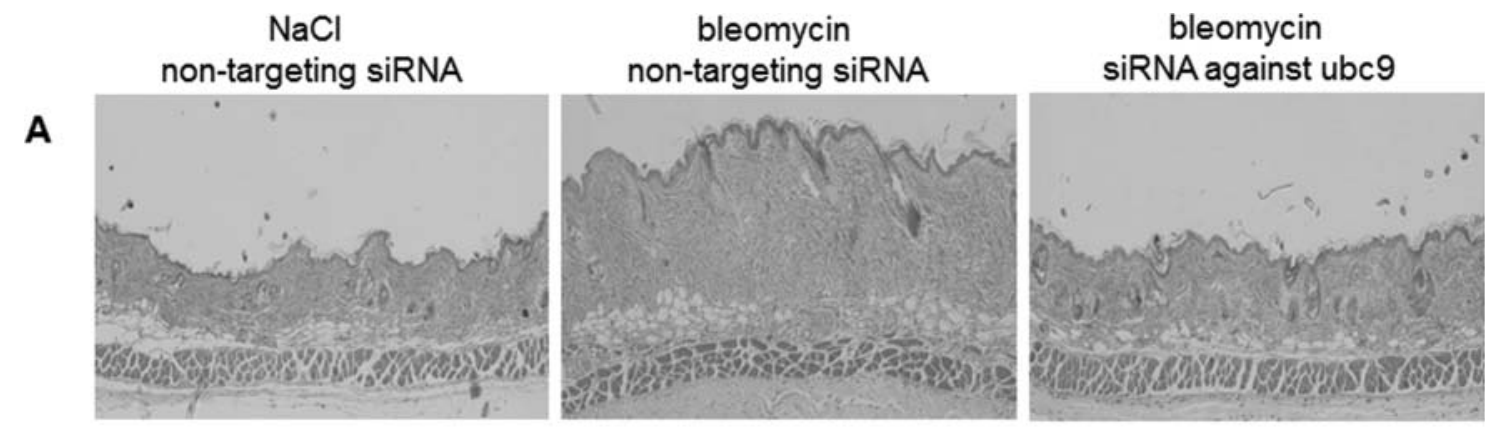

\section{$\mathrm{NaCl}+$ n.t. siRNA $\square$ bleomycin + n.t. siRNA $\square$ bleomycin + ubc9 siRNA}

B

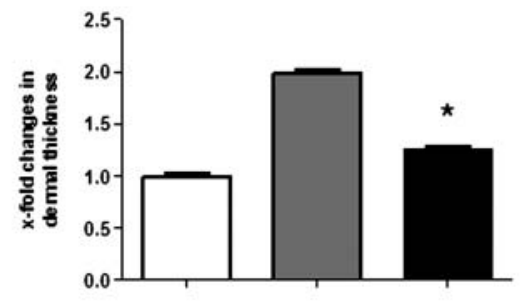

D

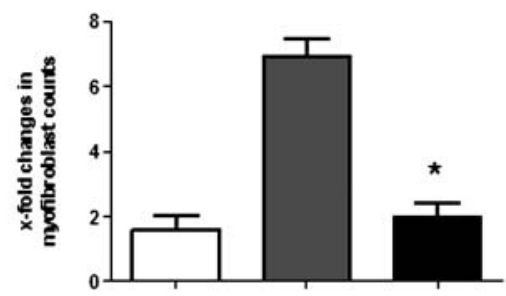

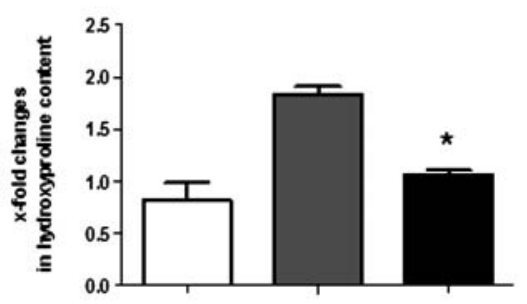

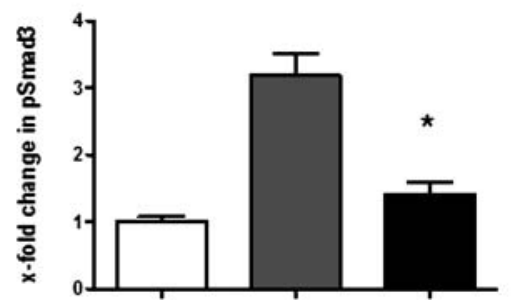

Figure 2 Inhibition of sumoylation almost completely prevents bleomycin-induced dermal fibrosis. (A) Representative sections of mice challenged with bleomycin and injected with Ubc9 siRNA $(n=4)$, mice injected with bleomycin and non-targeting control siRNA ( $n=4)$, and control animals injected with $\mathrm{NaCl}$ and non-targeting control siRNA $(n=4)$ are shown at 100 -fold magnification. White bars indicate the dermal thickness.

(B) Decreased dermal thickening in bleomycin-challenged mice injected with siRNA against Ubc9, compared with bleomycin plus non-targeting siRNA. Reduced hydroxyproline content (C) and decreased myofibroblast counts (D) upon inhibition of sumoylation. (E) Knockdown of Ubc9 prevents the nuclear accumulation of phosphorylated Smad3 upon challenge with bleomycin. n.t. siRNA: non-targeting siRNA. ${ }^{*} p<0.05$, As compared with bleomycin-challenged, sham-treated mice. 

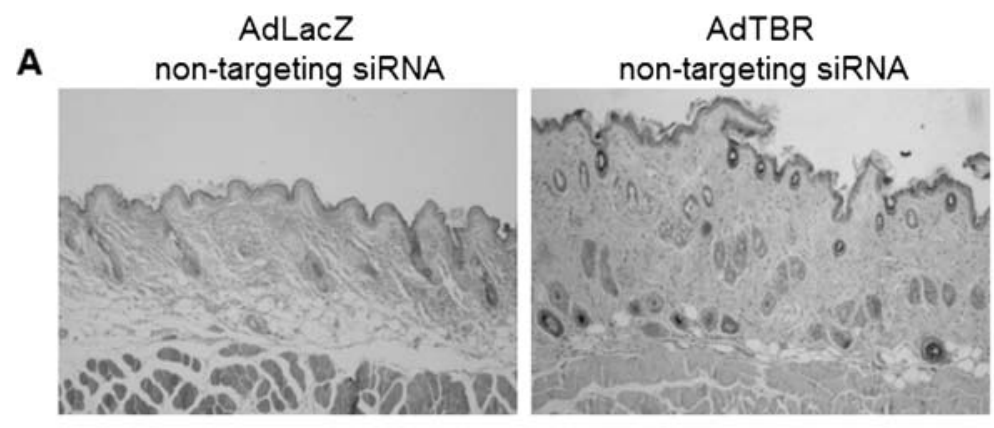

\section{siRNA against ubc9}

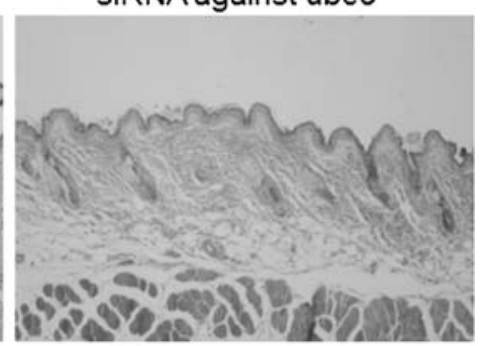

$A d L a c Z+n . t . ~ s i R N A$

B

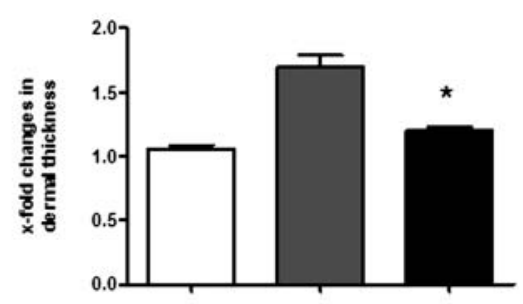

D

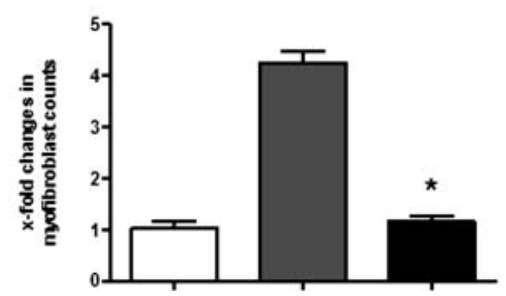

AdTBR+ n.t. siRNA
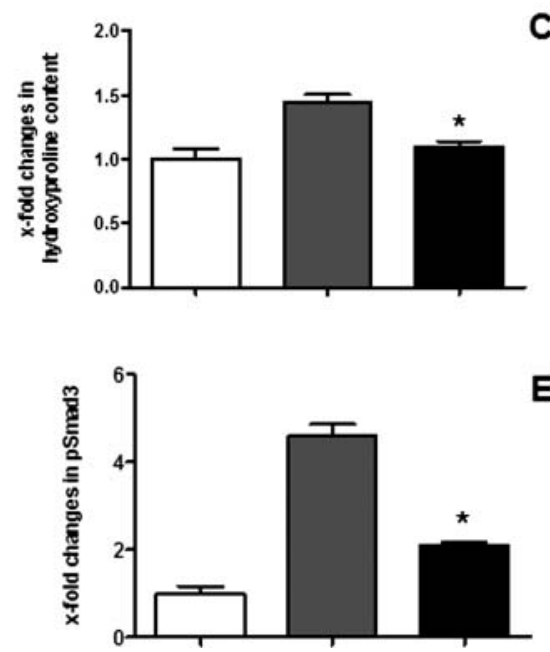

Figure 3 siRNA-Mediated knockdown of Ubc9 reduces TGF- $\beta$ receptor (TBR) induced fibrosis. (A) Representative sections of mice injected with AdLacZ and control siRNA ( $n=5)$, active TGF- $\beta$ receptor type I (AdTBR) and control siRNA $(n=5)$, and AdTBR plus siRNA against Ubc9 $(n=5)$ are shown at 100-fold magnification. White bars indicate the dermal thickness. Inhibition of sumoylation prevents dermal thickening (B), decreases the hydroxyproline content (C) and reduces the differentiation of resting fibroblasts into myofibroblasts (D). (E) Knockdown of Ubc9 decreases the nuclear accumulation of phosphorylated Smad3 induced by overexpression of TBR. n.t. siRNA: non-targeting siRNA. ${ }^{*} p<0.05$ As compared with bleomycin-challenged, sham-treated mice.

sumoylation may target TGF- $\beta$ signalling. Consistently, siRNA against Ubc9 prevented the nuclear accumulation of phosphorylated Smad3, confirming decreased canonical TGF- $\beta$ / Smad signalling. Indeed, evidence from in vitro studies indicates that several components of the canonical TGF- $\beta / S m a d$ pathway, such as TBR type $1, \mathrm{Smad} 3$ and Smad4 are substrate to sumoylation. ${ }^{10-12}$ However, the functional outcomes on canonical TGF $\beta / S m a d$ pathway reported in these studies were conflicting. Sumoylation of TGF $\beta$ receptor type 1 and Smad4 stimulated TGF- $\beta /$ Smad signalling by increasing the recruitment of receptor Smads and enhancing their stability, respectively. ${ }^{11} 1219$ On the other hand, sumoylation of Smad3 and Smad4 were reported to inhibit canonical TGF- $\beta /$ Smad signalling by stimulating the nuclear export and decreasing the transcriptional activity. ${ }^{10} 20$ Although we did not analyse the individual components of the TGF- $\beta / S$ mad pathway, and further mechanistic studies are crucial to extending our understanding on the role of sumoylation in the pathogenesis of SSc, our results demonstrate, in an extension of previous in vitro studies, that general inhibition of sumoylation dampens canonical TGF- $\beta /$ Smad signalling. Given the key role of TGF- $\beta$ signalling in other fibrotic diseases, targeting of sumoylation maybe a potential therapeutic approach in other fibrotic disorders. However, further studies are required to confirm the effect of sumoylation in other fibrotic conditions. However, besides regulating canonical TGF- $\beta / S$ mad signalling, sumoylation may also contribute to the pathogenesis of SSc on other levels, for example, by regulating the catalytic activity of topoisomerase I, or by enhancing canonical Wnt signalling. ${ }^{8}$

In summary, we demonstrated that sumoylation is increased in SSc, and may contribute to fibrosis in SSc. Inhibition of sumoylation by knockdown of Ubc9 almost completely prevented the development of fibrosis and inhibited canonical TGF- $\beta /$ Smad signalling in experimental models, indicating a key role of sumoylation in the pathogenesis of SSc.

Acknowledgements We thank Anna-Maria Herrmann, Maria Halter, Madeleine Demleitner, Verena Wäsch and Stefan Fritz for excellent technical assistance.

Contributors Design of the study: $A D, O D, G S$, JHWD; acquisition of data: $A K, A D$, $C D, V L, C B, K G$; interpretation of data: $A H, A D, C D$, JHWD; manuscript preparation: $A K, C D, C B, J H W D$; providing essential material: $H S$.

Funding Grants DI 1537/2-1, DI 1537/4-1, DI 1537/5-1, AK 144/1-1 and SCHE $1583 / 7-1$ of the Deutsche Forschungsgemeinschaft, grants A20 and A40 of the IZKF in Erlangen, the ELAN-Program of the University of Erlangen-Nuremberg (to VL and $\mathrm{CB})$, and the Career Support Award of Medicine of the Ernst Jung Foundation (to JHWD).

\section{Competing interests None.}

Ethics approval Ethical Committee of the University of Erlangen-Nuremberg.

Provenance and peer review Not commissioned; externally peer reviewed. 


\section{REFERENCES}

1. Gabrielli A, Avvedimento EV, Krieg T. Scleroderma. N Engl J Med 2009;360:1989-2003.

2. Varga J, Abraham D. Systemic sclerosis: a prototypic multisystem fibrotic disorder J Clin Invest 2007;117:557-67.

3. Huber LC, Stanczyk J, Jungel A, et al. Epigenetics in inflammatory rheumatic diseases. Arthritis Rheum 2007:56:3523-31.

4. Wilkinson KA, Henley JM. Mechanisms, regulation and consequences of protein SUMOylation. Biochem J 2010;428:133-45.

5. Wang Y, Dasso M. SUMOylation and deSUMOylation at a glance. J Cell Sci 2009;122:4249-52.

6. Driscoll JJ, Dechowdhury R. Therapeutically targeting the SUMOylation, Ubiquitination and Proteasome pathways as a novel anticancer strategy. Target Oncol 2010;5:281-89.

7. Huber LC, Distler JH, Moritz F, et al. Trichostatin a prevents the accumulation of extracellular matrix in a mouse model of bleomycin-induced skin fibrosis. Arthritis Rheum 2007;56:2755-64.

8. Zhou X, Lin W, Tan FK, et al. Decreased catalytic function with altered sumoylation of DNA topoisomerase I in the nuclei of scleroderma fibroblasts. Arthritis Res Ther 2011;13:R128.

9. Jungel A, Distler JH, Gay S, et al. Epigenetic modifications: novel therapeutic strategies for systemic sclerosis? Expert Rev Clin Immunol 2011;7:475-80.

10. Imoto S, Ohbayashi N, Ikeda 0, et al. Sumoylation of Smad3 stimulates its nuclear export during PIASy-mediated suppression of TGF-beta signaling. Biochem Biophys Res Commun 2008;370:359-65.
11. Kang JS, Saunier EF, Akhurst RJ, et al. The type I TGF-beta receptor is covalently modified and regulated by sumoylation. Nat Cell Biol 2008;10:654-64.

12. Lin $\mathbf{X}$, Liang $M$, Liang $Y$, et al. Activation of transforming growth factor-beta signaling by SUMO-1 modification of tumor suppressor Smad4/DPC4. J Biol Chem 2003;278:18714-19.

13. LeRoy EC, Black C, Fleischmajer R, et al. Scleroderma (systemic sclerosis): classification, subsets and pathogenesis. J Rheumatol 1988;15:202-5.

14. Horn A, Kireva T, Palumbo-Zerr K, et al. Inhibition of hedgehog signaling prevents experimental fibrosis and induces regression of established fibrosis. Ann Rheum Dis 2012;71:785-9.

15. Palumbo K, Zerr P, Tomcik M, et al. The transcription factor JunD mediates transforming growth factor $\{$ beta\}-induced fibroblast activation and fibrosis in systemic sclerosis. Ann Rheum Dis 2011;70:1320-6.

16. Akhmetshina A, Palumbo-Zerr K, Dees C, et al. Activation of canonical Wnt signalling is required for TGF- $\beta$ mediated fibrosis. Nature Commun 2012;13:735.

17. Beyer C, Reich N, Schindler SC, et al. Stimulation of soluble guanylate cyclase reduces experimental dermal fibrosis. Ann Rheum Dis 2012;71:1019-26.

18. Beyer C, Schramm A, Akhmetshina A, et al. beta-catenin is a central mediator of pro-fibrotic Wnt signaling in systemic sclerosis. Ann Rheum Dis 2012;71:761-7.

19. Lee PS, Chang C, Liu D, et al. Sumoylation of Smad4, the common Smad mediator of transforming growth factor-beta family signaling. J Biol Chem 2003;278:27853-63.

20. Long J, Wang G, He D, et al. Repression of Smad4 transcriptional activity by SUMO modification. Biochem J 2004;379:23-9. 


\section{ARD Inhibition of sumoylation prevents experimental fibrosis}

Aisa Khodzhigorova, Alfiya Distler, Veronika Lang, Clara Dees, Holm Schneider, Christian Beyer, Kolja Gelse, Oliver Distler, Georg Schett and Jörg H W Distler

Ann Rheum Dis 2012 71: 1904-1908 originally published online August 17,2012

doi: 10.1136/annrheumdis-2012-201746

Updated information and services can be found at:

http://ard.bmj.com/content/71/11/1904

These include:

References This article cites 20 articles, 8 of which you can access for free at: http://ard.bmj.com/content/71/11/1904\#BIBL

Email alerting service

Receive free email alerts when new articles cite this article. Sign up in the box at the top right corner of the online article.

ErrataAn erratum has been published regarding this article. Please see next page or:

http://ard.bmj.com/content/72/6/1110.1.full.pdf

Topic Articles on similar topics can be found in the following collections

Collections

Connective tissue disease (4234)

\section{Notes}

To request permissions go to:

http://group.bmj.com/group/rights-licensing/permissions

To order reprints go to:

http://journals.bmj.com/cgi/reprintform

To subscribe to BMJ go to:

http://group.bmj.com/subscribe/ 


\section{Corrections}

Khodzhigorova A, Distler A, Lang V, et al. Inhibition of sumoylation prevents experimental fibrosis. Ann Rheum Dis 2012;71:1904-8. In panel A of figure 3 there are three histological images. The image on the left is from AdLacZ control mouse treated with a non-targeting siRNA, while the image on the right is from a genetically modified AdTBR mouse treated with siRNA against ubc9. However, the two histological pictures are identical to each other. The correct image of AdLacZ control mice treated with non-targeting siRNA is shown below.
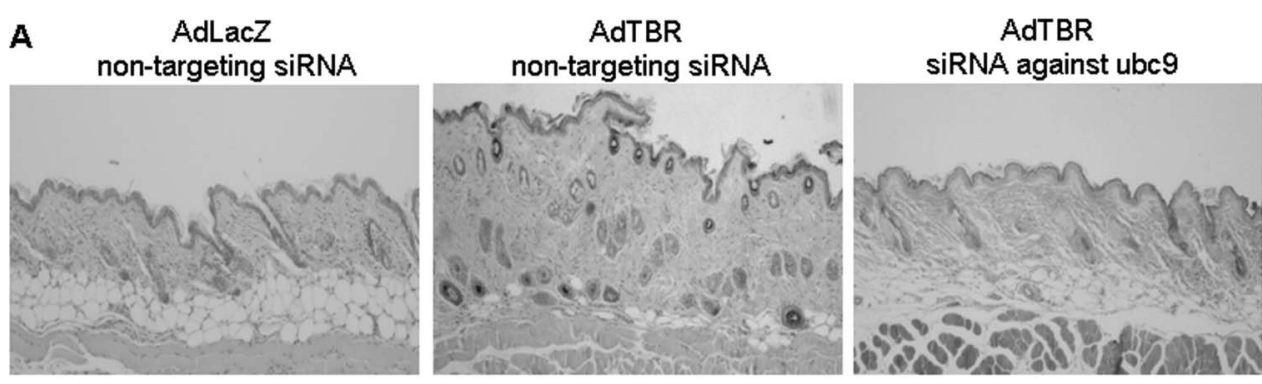

AdLacZ + n.t. siRNA

$\square$ AdTBR+ n.t. SiRNA

AdTBR + ubc9 siRNA

B
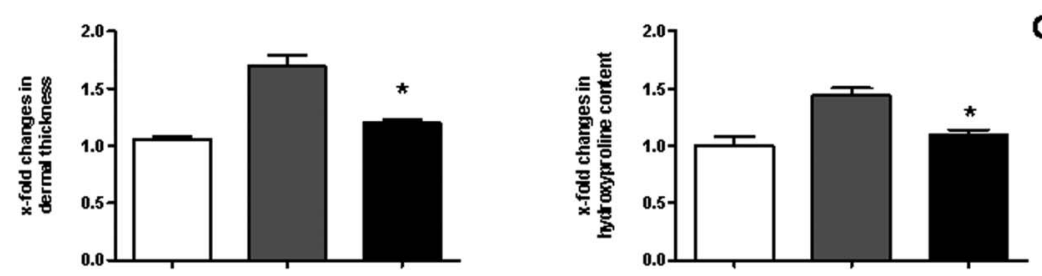

D
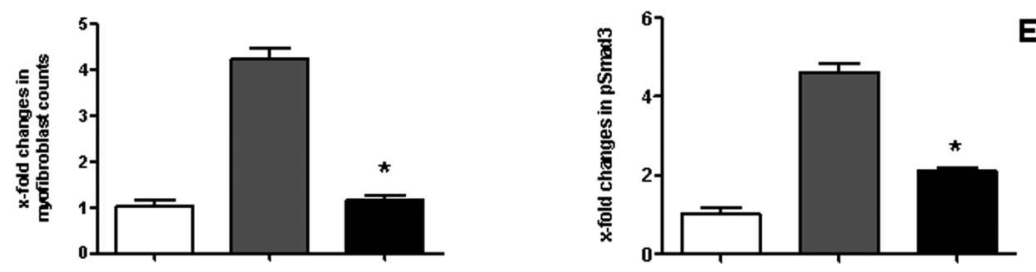

Ann Rheum Dis 2013;72:1110. doi:10.1136/annrheumdis-2012-201746corr1 